\title{
A Local Distance Feature Extraction Based Shortwave Direction Finding Cross Location Algorithm
}

\author{
Xi Shen ${ }^{1, a}$, Jie Zhu ${ }^{2, b^{*}}$, Dayong Liu $^{1, a}$ and Xiaofei Zhang ${ }^{1, \text { a }}$ \\ ${ }^{1}$ No.80 Beilishi Road, Xicheng District, Beijing, P.R.China \\ ${ }^{2}$ No.8 Tongji Nanlu, Beijing Economic Technology Development Area, P.R. China \\ a shenxi@srrc.org.cn, ${ }^{\mathrm{b}}$ zhujie@cesi.cn (corresponding author)
}

\begin{abstract}
Keywords: shortwave location algorithm, local feature extraction, direction finding.
Abstract. This paper innovatively proposes a local distance feature based shortwave direction finding cross location algorithm. The proposed algorithm is accomplished by setting one principle direction finding station and extracting the local distance feature in the first localization results. The azimuth subset is created considering the local distance feature and the principle direction finding station. The measured results show that compared to the traditional cross location algorithm, the proposed local distance feature extraction based location algorithm increases the location accuracy and decrease the location error.
\end{abstract}

\section{Introduction}

Localization one shortwave emitter plays a vital role in military uses [1]. Over the last few years, many researchers have devoted their efforts to the localization methods of shortwave emitters. Three main categories are divided in the shortwave emitter localization methods [2-6], i.e. direction of arrival based method (DOA), time difference of arrival based method (TDOA) and direction and time difference of arrival based method. The estimation of the arrival time needs very strict time synchronization. But it is not the same thing for DOA based method. Over the few years, the DOA based localization method has been applied in large-scale engineering fields due to its low system complexity requirements. Many researches have been done to increase the localization accuracy by processing the DOA parameter [7-9].

But the DOA based localization system has much lower accuracy than that of the other two method based localization system. Increasing the localization accuracy is an urgent issue. The weighted average method, the least square method, the maximum likelihood method, the second feature extraction based method are usually used in the location technology to increase the location accuracy [10-13]. But due to the geographic distribution of the monitoring stations of the state radio monitoring center, much more monitoring stations are concentrated in the east area than that in the west area. And the localization error of the emitters in the west is much bigger than that in the east. The up mentioned localization method do not considering the distribution factor and have bigger error to locate the emitters in the west. As known to our knowledge, it is the first time that the proposed algorithm takes the distribution of the monitoring stations into consideration. It is accomplished by setting one principle monitoring station and extracting the local distance feature in the first localization results. The azimuth subset is created considering the local distance feature and the principle station. The measured results show compared to the traditional cross location algorithm, the proposed one not only increases the location accuracy but also improves the performance of the shortwave location system.

\section{Problem Formulation}

Assume that the shortwave direction finding cross location system is composed of $\mathrm{m}$ direction finding stations (i.e. DF station) with the coordinates DFn (Xi,Yi) (i=1, 2, 3, .., m) respectively [13]. And the azimuth of each DF station is $\theta \mathrm{i}(\mathrm{i}=1,2,3, \ldots, \mathrm{m})$ respectively. In the absence of noise, the DF lines will intersect at a single point. Taking the influence of ionosphere changes into consideration, the errors in azimuth cannot be inevitable. This errors which is represented by $\Delta \theta \mathrm{i}(\mathrm{i}=1,2,3, \ldots, \mathrm{m})$ 
for each DF station will create a polygon-shaped area instead of a single point. And the target location of the emitter is within this polygon-shaped area. Here three DF stations are taken for example as shown in Fig.1. Considering the errors of azimuth, the target location is represented by the polygon-shaped area with thick line. Regardless of these errors, the target location is a single point represented by $\star$. It is worth noting that the number of DF stations and their geographical position distribution will influence the polygon-shaped area and then influence the final target location of emitter. In practical application, more than three DF stations are used to locate the target emitter. And in the situation with more than three DF stations, there would be much more intersections. Here the method applied to the situation with three DF stations can be applied to other situations.

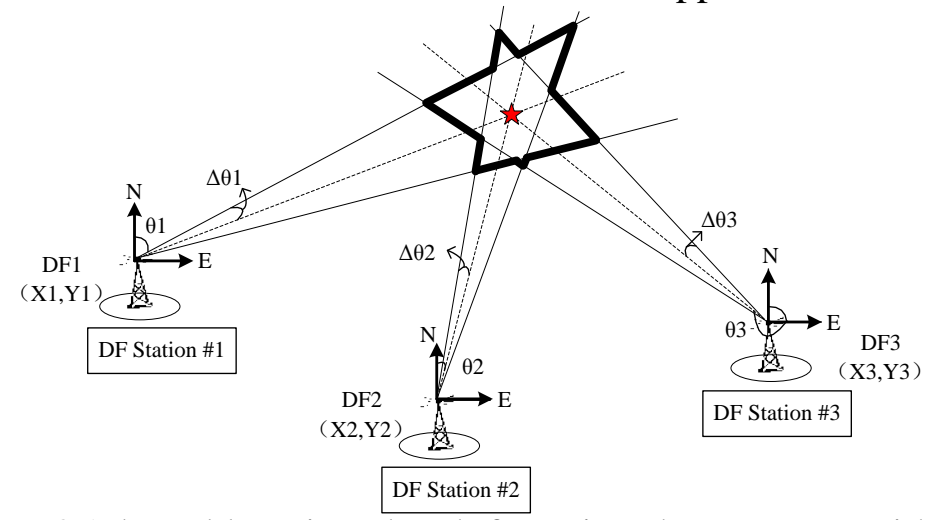

Fig. 1 DOA based location sketch for azimuth error case with $3 \mathrm{DF}$

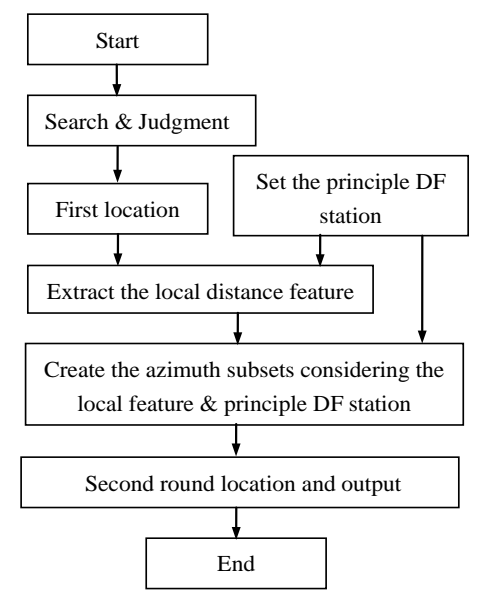

Fig. 2 The flowchart of the proposed local distance feature extraction based localization algorithm

\section{Local Distance Feature Extraction Based Shortwave Direction Finding Cross Location Algorithm}

In this section, a local distance feature extraction based shortwave direction finding cross location algorithm is proposed. The basic idea of the proposed algorithm is to take the geographic distribution of the monitoring stations into consideration. And by setting one principle direction finding station and extracting the local feature in the first location results, the azimuth subset is been created. The algorithm flowchart is presented in Fig.2. The main algorithm steps are as follows:

(1) Get the first location results [13].

Assume the number of direction finding stations is $\mathrm{m}$. In this step, the first location results are gotten and would be used in the next step. Usually, we can use the traditional shortwave emitter direction finding cross location algorithm to get the first location results. Here the weight of azimuth lines level $\delta_{j}$ and the weight of crossing angle level $\alpha_{j k}$ are introduced to define the reliability of measured azimuths and crossing angles. The final target location is determined by these two weight variables. The best estimate latitude coordinates for several direction finding stations can be calculated by Eq. (1) and Eq. (2). 


$$
\begin{gathered}
X_{T}=\frac{\sum_{j, k=1(j \neq k)}^{m}\left(\mathrm{X}_{T_{j k}} \frac{1}{\delta_{j} \delta_{k}} \frac{1}{\alpha_{j k}}\right)}{\sum_{j, k=1(j \neq k)}^{m}\left(\frac{1}{\delta_{j} \delta_{k}} \frac{1}{\alpha_{j k}}\right)} \\
Y_{T}=\frac{\sum_{j, k=1(j \neq k)}^{m}\left(\mathrm{Y}_{T_{j k}} \frac{1}{\delta_{j} \delta_{k}} \frac{1}{\alpha_{j k}}\right)}{\sum_{j, k=1(j \neq k)}^{m}\left(\frac{1}{\delta_{j} \delta_{k}} \frac{1}{\alpha_{j k}}\right)}
\end{gathered}
$$

(2) Set the principle DF station.

In this step, one DF station is set as the principle station based on our experience. In general, the DF station in your work place is chosen.

(3) Extraction the local distance feature.

Process the first location results to get the distance feature. It is noted that the azimuth of the principle DF station is not considered. We extracted the azimuths from the other DF stations.

Calculate the great-circle distance between the first location result $\left(\mathrm{X}_{\mathrm{T}}, \mathrm{Y}_{\mathrm{T}}\right)$ and $\mathrm{m}-1$ direction finding stations respectively. And the measurement distance matrix $D$. The ${ }^{D_{i}}$ means the great-circle distance between the first location results $\left(\mathrm{X}_{\mathrm{T}}, \mathrm{Y}_{\mathrm{T}}\right)$ and the $\mathrm{i}^{\text {th }}$ direction finding station.

Set the threshold value $k_{1}$ and $k_{2}$ in kilometers. If $k_{1}<D_{i}<k_{2}$, the corresponding direction finding station is available. Choose two available direction finding stations (i.e. DF station A and DF station B) which have the less two great-circle distance difference. This is the local distance feature.

(4) Create the azimuth subsets.

In this step, the azimuth subsets are created. It is composed of the azimuth of the principle DF station and these of DF station A and DF station B.

(5) Second round location and output.

The final results are gotten using the azimuth subsets in step (4).

The proposed algorithm extracted the local distance feature. And create the azimuth subsets by considering the local feature and principle station. The azimuth subsets are used in the second round location.

These threshold values in the algorithm debug are as shown in Table 1.

Table 1. Parameters of the proposed algorithm

\begin{tabular}{cll}
\hline Parameters & Meaning & Values \\
\hline$\delta_{j}$ & Weight of azimuth line level & - \\
$\alpha_{j k}$ & Weight of crossing angel level & - \\
$k_{1}$ & Threshold \#1 & $700[\mathrm{~km}]$ \\
$k_{2}$ & Threshold \#2 & $2000[\mathrm{~km}]$ \\
\hline
\end{tabular}

\section{Measurement Results and Discussions}

To minimize the influence of the ionosphere changes and the man-made influence, we permit that these azimuths of different frequency signals for the same emitter are gotten at the same time every day for each DF station by a certain worker. And the measurements last several months to get adequate data.

Here No.1 emitter with the coordinate which located at $108.61^{\circ}$ east longitude and $34.37^{\circ}$ north latitude and No.2 emitter with the coordinate which located at $91.15^{\circ}$ east longitude and $29.63^{\circ}$ north latitude are taken for example. The emitters are all located in the west region of our country. The distribution of the measured location results gotten from the proposed algorithm and the traditional 
shortwave direction finding cross location algorithm is shown in Fig.3.(a) and Fig.4.(a) corresponding to these two mentioned emitters.

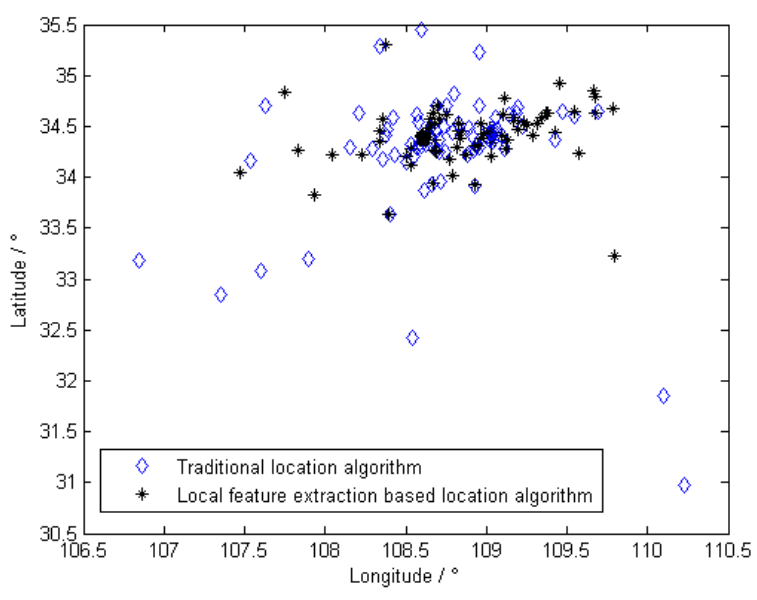

(a)

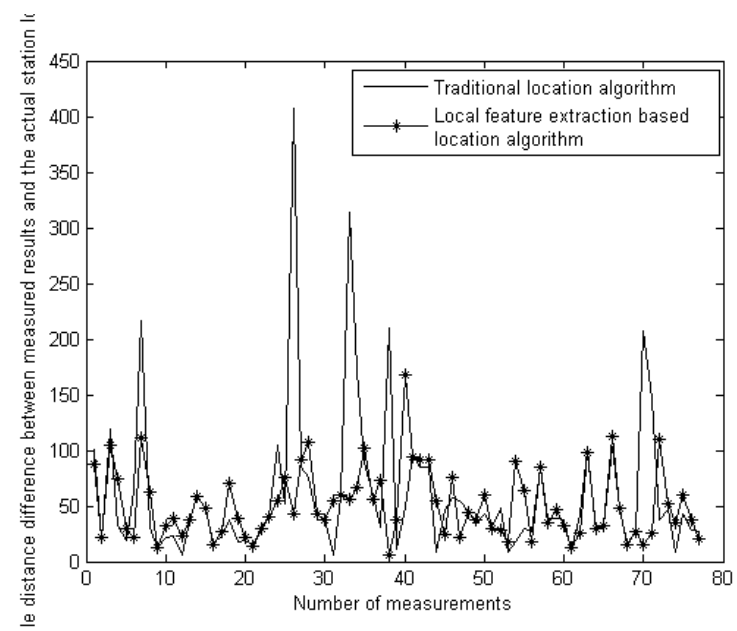

(b)

Fig.3. Results of No.1 emitter (a) The distribution of the measured location results gotten from the proposed algorithm and the traditional algorithm; (b) The great-circle difference between the measured results and the actual location of the emitter

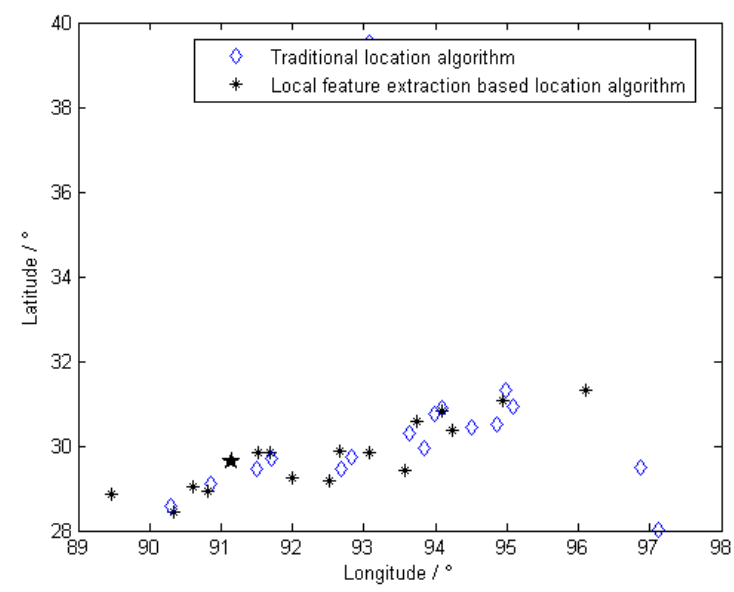

(a)

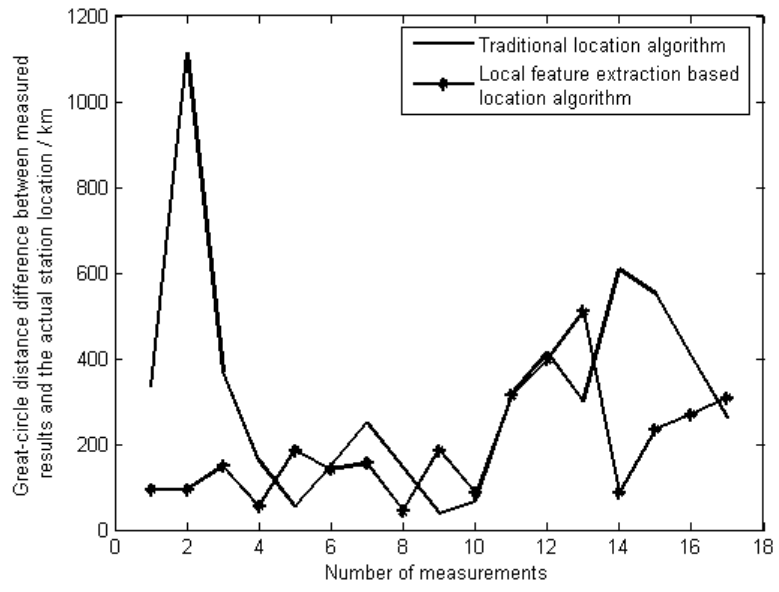

(b)

Fig.4. Results of No.2 emitter (a) The distribution of the measured location results gotten from the proposed algorithm and the traditional algorithm; (b) The great-circle difference between the measured results and the actual location of the emitter

The shape $\star$ represents the actual geographical location of the target emitter. It is clearly that the measured location results gotten from the proposed algorithm are much more concentrated around the actual location of the target emitter than that gotten from the traditional algorithm.

The great-circle difference between the measured result and the actual location of the emitter is shown in Fig.3.(b) and Fig.4.(b). It is clearly on the whole, the great-circle difference between the measured result and the actual location of the emitter for the proposed algorithm is much lower than that for the traditional algorithm.

In Fig.3.(b), the numerical results show that maximum great-circle difference for the traditional algorithm is about $407 \mathrm{~km}$, and that for the proposed algorithm is about $168 \mathrm{~km}$. The RMS error of measured results for the traditional algorithm is about $90.5 \mathrm{~km}$, and that for the proposed algorithm is just about $60.1 \mathrm{~km}$. There is apparently a 33.6\% drop in the RMS error. In Fig.4.(b), the RMS error of measured results for the traditional algorithm is about $412.8 \mathrm{~km}$, and that for the proposed algorithm is just about $231.2 \mathrm{~km}$. There is apparently a $43.99 \%$ drop in the RMS error.

So the quantified measured results illustrate that compared to the traditional cross algorithm, the proposed algorithm increase the location accuracy and decrease the location error especially for the emitters located in the west region. 


\section{Summary}

In this paper, a local distance feature extraction based shortwave direction finding location algorithm is proposed to increase the location accuracy of the current shortwave location system. The proposed algorithm is accomplished by setting one principle direction finding station and extracting the local feature in the first location results. Then the azimuth subsets are created taken the principle DF station and the local distance feature into consideration. The quantified measurements illustrate that the proposed local distance feature extraction based shortwave direction finding cross location algorithm can decrease the localization error and improve the performance of the current shortwave localization system.

\section{Acknowledgement}

This work was supported in part by the National Natural Science Foundation of China (Grant No.61227801).

\section{References}

[1] Poisel, R.A. Electronic warfare target location methods. Boston: Artech House, 2005.

[2] Stansfield, R.G. Statistical theory of DF fixing. Journal of IEEE 14 part IIIA(15), 1947, pp: 762-770.

[3] Gavish, M. \& Weiss, A. J. Performance analysis of bearing-only target location algorithms, IEEE Transactions on Aerospace and Electronic Systems, 1992, Vol. 28, No.3, pp: 817-828.

[4] Torrieri, D.J. Statistical theory of passive location systems. IEEE Transactions on Aerospace and Electronic Systems , 1984, Vol. 20, No.2, pp: 183-198.

[5] Dogancay, K. \& Gray, D. A. Closed-form estimators for multi-pulse TDOA localization, Proceedings of the Eighth International Symposium on Signal Processing and Its Applications, 2005, Vol. 2, pp: 543-546.

[6] Jianliang, S. Mueller, H. C. Marx, M. Pedestrian detection and localization using antenna array and sequential triangulation, IEEE Proceedings of Intelligent Transportation Systems, 2005, pp: 126-130.

[7] Chen, L. Study of passive location and tracking technique. Beijing: Doctoral Dissertation of Beijing Uni-versity of Aeronautics and Astronautics (in Chinese), 2002.

[8] Fu, X. The design and application of direction finding and location in the national wireless shortwave monitoring system. Sichuan: Master Dissertation of Sichuan University(in Chinese), 2006.

[9] Abdual Matheen, S. Padam Raju, K. Murali Prasad, G. Location fixing by 3 DF stations using triangulation method. International Journal of Research in Computer and Communication Technology, 2012, Vol. 1, No. 3, pp: 75-81.

[10] Xu, J.R. \& Xue, L. LS algorithm used in DF and location. Chinese Journal of Radio Science, 2001, Vol. 16, No.2, pp: 227-230. (in Chinese)

[11] Liu, J.J. Gong, X.F. Yang, J.J. Du, L. Algorithm re-search based on DF and location. The modern electronic technology, 2004, Vol. 171, No.4, pp: 49-55. (in Chinese)

[12] Song, W.B. \& Ma, X. A new target location algorithm with bearing-only measurements. Telecommunication engineering, 2014, Vol. 54, No.11, pp: 1488-1492. (in Chinese)

[13] Shen, X. Liu, R. Ji, W.L. Liu, D.Y. Zhang, X.F. A second-feature extraction based high frequency emitter direction finding cross location algorithm. $20142^{\text {nd }}$ International Conference on Systems and Informatics (ICSAI 2014), 2014. 